Article

\title{
Continuous Dependence of Solutions of Integer and Fractional Order Non-Instantaneous Impulsive Equations with Random Impulsive and Junction Points
}

\author{
Yu Chen ${ }^{1}$ and JinRong Wang ${ }^{1,2, *(D)}$ \\ 1 Department of Mathematics, Guizhou University, Guiyang 550025, China; cyrainie@126.com \\ 2 School of Mathematical Sciences, Qufu Normal University, Qufu 273165, China \\ * Correspondence: jrwang@gzu.edu.cn
}

Received: 19 February 2019; Accepted: 28 March 2019; Published: 4 April 2019

\begin{abstract}
This paper gives continuous dependence results for solutions of integer and fractional order, non-instantaneous impulsive differential equations with random impulse and junction points. The notion of the continuous dependence of solutions of these equations on the initial point is introduced. We prove some sufficient conditions that ensure the solutions to perturbed problems have a continuous dependence. Finally, we use numerical examples to demonstrate the obtained theoretical results.
\end{abstract}

Keywords: non-instantaneous impulsive equations; random impulsive and junction points; continuous dependence

MSC: 34A37; 34A08

\section{Introduction}

Impulsive differential equations (IDEs) are applied in many fields, such as mechanical engineering, biology, and medical science. Generally speaking, there are two classes of impulsive equations. One is composed of instantaneous IDEs, for which the duration of the impulsive perturbation is very short compared to the entire evolution process, see for example, References [1,2]. The other class is composed of non-instantaneous IDEs, for which the impulsive action starts at a fixed point, and remains active over a period of time that may be related to the previous state.

Non-instantaneous IDEs were introduced in Reference [3] and address the shortcomings of instantaneous IDEs, which do not seem to describe some of the dynamics of evolution in pharmacotherapy. Wang and Fečkan [4] corrected non-instantaneous impulsive equations in Reference [3] and proposed new and generalized non-instantaneous IDEs by considering the impact from the previous system state. Wang [5] used the notion of a non-instantaneous impulsive operator to represent the solutions of linear problems, which are simplified from the model in Reference [4]. The existence and stability of solutions and control problems for these non-instantaneous IDEs, as well as inclusions have been studied in References [6-25]. Meanwhile, fractional differential equations provide an alternative model and are gaining much importance and attention. The qualitative theory of fractional differential equations was studied extensively in the literature; see References [26-33] and the references therein.

Recently, Dishlieva [34] studied a class of instantaneous IDEs with random impulsive effects and established sufficient conditions to ensure continuous dependence of the solutions. Motivated by 
Reference [34], we investigate the continuous dependence of the solutions of the following first-order nonlinear differential equations with random non-instantaneous impulsive effects:

$$
\left\{\begin{array}{l}
x^{\prime}(t)=\mathbf{f}(t, x(t)), \quad t \in\left(s_{i}, t_{i+1}\right], i \in \mathbf{N}:=\{0,1,2, \cdots\}, \\
x\left(t_{i}^{+}\right)=\mathbf{h}_{\mathbf{i}}\left(t_{i}, x\left(t_{i}^{-}\right)\right), \quad i \in \mathbf{N}^{+}:=\{1,2, \cdots\}, \\
x(t)=\mathbf{h}_{\mathbf{i}}\left(t, x\left(t_{i}^{-}\right)\right), \quad t \in\left(t_{i}, s_{i}\right], i \in \mathbf{N}^{+}, \\
x(0)=x_{0},
\end{array}\right.
$$

and also of the fractional-order random non-instantaneous IDEs:

$$
\left\{\begin{array}{l}
{ }^{C} \mathbf{D}_{s_{i},}^{\alpha} x(t)=\mathbf{f}(t, x(t)), \quad t \in\left(s_{i}, t_{i+1}\right], i \in \mathbf{N}, \alpha \in(0,1), \\
x\left(t_{i}^{+}\right)=\mathbf{h}_{\mathbf{i}}\left(t_{i}, x\left(t_{i}^{-}\right)\right), \quad i \in \mathbf{N}^{+}, \\
x(t)=\mathbf{h}_{\mathbf{i}}\left(t, x\left(t_{i}^{-}\right)\right), \quad t \in\left(t_{i}, s_{i}\right], i \in \mathbf{N}^{+}, \\
x(0)=x_{0},
\end{array}\right.
$$

where ${ }^{C} \mathbf{D}_{s_{i}, t}^{\alpha}$ denotes the classical Caputo fractional derivative of order $\alpha$, by changing the lower limit $s_{i}$, as in Reference [35]. The random impulse and junction points, $t_{i}$ and $s_{i}$, respectively, satisfy $t_{0}=s_{0}=0<t_{1}<s_{1}<t_{2}<\cdots<s_{i}<t_{i+1}<\cdots, t_{i} \rightarrow \infty$. The symbol $x\left(t_{i}^{+}\right)$and $x\left(t_{i}^{-}\right)$ represent the right and left limits of $x(t)$ at $t=t_{i}$, respectively. In addition, we set $x\left(t_{i}^{-}\right)=x\left(t_{i}\right)$. The function $\mathbf{f}:[0, \infty) \times \mathbf{R} \rightarrow \mathbf{R}$ is continuous, and the function $\mathbf{h}_{\mathbf{i}}:\left[t_{i}, s_{i}\right] \times \mathbf{R} \rightarrow \mathbf{R}$ is continuous for all $i \in \mathbf{N}^{+}$. The piecewise continuous solutions of Equations (1) and (2) have been represented in Reference [14] [Equations (5) and (7), therein].

We also introduce the following related original and perturbed equations without impulses:

$$
\begin{gathered}
\left\{\begin{array}{l}
X^{\prime}(t)=\mathbf{f}(t, X(t)), t \in\left[s_{i}, t_{i+1}\right], i \in \mathbf{N}, \\
X\left(s_{i}\right)=x_{s_{i}},
\end{array}\right. \\
\left\{\begin{array}{l}
X^{\prime}(t)=\mathbf{f}(t, X(t)), t \in\left[s_{i}, t_{i+1}\right], i \in \mathbf{N}, \\
X\left(s_{i}\right)=\widetilde{x}_{s_{i}},
\end{array}\right. \\
\left\{\begin{array}{l}
{ }^{C} \mathbf{D}_{s_{i},}^{\alpha} X(t)=\mathbf{f}(t, X(t)), t \in\left[s_{i}, t_{i+1}\right], i \in \mathbf{N}, \alpha \in(0,1), \\
X\left(s_{i}\right)=x_{s_{i}},
\end{array}\right.
\end{gathered}
$$

and:

$$
\left\{\begin{array}{l}
{ }^{C} \mathbf{D}_{s_{i}, t}^{\alpha} X(t)=\mathbf{f}(t, X(t)), t \in\left[s_{i}, t_{i+1}\right], i \in \mathbf{N}, \alpha \in(0,1), \\
X\left(s_{i}\right)=\widetilde{x}_{s_{i}} .
\end{array}\right.
$$

Denote any solution of Equations (1) or (2) by $x\left(\cdot ; 0, x_{0}\right) \in P C(\mathbf{J}, \mathbf{R}):=\{x: \mathbf{J} \rightarrow \mathbf{R}: x \in$ $\mathbf{C}\left(\left(t_{k}, t_{k+1}\right], \mathbf{R}\right), k=0,1, \cdots$ and there exists $x\left(t_{k}^{+}\right)$and $x\left(t_{k}^{-}\right), k=1,2, \cdots$ with $\left.x\left(t_{k}^{-}\right)=x\left(t_{k}\right)\right\}$, where $\mathbf{J}=[0, \infty)$ and $\mathbf{C}\left(\left(t_{k}, t_{k+1}\right], \mathbf{R}\right)$ denotes the space of all continuous functions from $\left(t_{k}, t_{k+1}\right]$ into $\mathbf{R}$. Additionally, denote any solution of Equations (3) or (5) by $X\left(\cdot ; s_{i}, x_{s_{i}}\right) \in \mathbf{C}\left(\left[s_{i}, t_{i+1}\right], \mathbf{R}\right)$. For the interval $\left(t_{i}, s_{i}\right]$, we denote its solutions by $X\left(\cdot ; t_{i}, x\left(t_{i}^{+}\right)\right)$. Then, the following relationship is valid:

$$
x\left(t ; 0, x_{0}\right)=\left\{\begin{array}{l}
X\left(t ; 0, x_{0}\right), t \in\left[0, t_{1}\right] \\
X\left(t ; t_{1}, x\left(t_{1}^{+}\right)\right), t \in\left(t_{1}, s_{1}\right] \\
X\left(t ; s_{1}, x_{s_{1}}\right), t \in\left(s_{1}, t_{2}\right] \\
\cdots \cdots
\end{array}\right.
$$

The main objective of this article is to present the continuous dependence of solutions with respect to the initial condition when random impulse and junction points are incorporated in Equations (1) and (2). We will take notice of the fact that the location and the number of the impulse points and junction points are not determined in a finite time interval, and so, we can assume that the impulse and junction points are random. 
The main contributions of this paper are two folds. We extend the concept and results in Reference [34] to random non-instantaneous impulsive cases by imposing different conditions on the nonlinear term. We also extend the continuous dependence of solutions of first-order non-instantaneous impulsive equations to fractional-order non-instantaneous impulsive equations.

The rest of this paper is organized as follows. Section 2 gives the relevant definitions and notions for the continuous dependence of solutions and contains the main results. Section 3 gives two examples to demonstrate the application of our results. In Section 4 , conclusions are drawn.

\section{Main Results}

Based on Reference [12] (Definition 2.1, therein) and Reference [34] (Definition 2, therein), we give the following definitions for the continuous dependence of solutions.

Definition 1. The solution $x\left(\cdot ; 0, x_{0}\right) \in P C(\mathbf{J}, \mathbf{R})$ of Equations (1) or (2) depends continuously on the initial point $\left(0, x_{0}\right)$, if for any $\varepsilon>0, \mathbf{T}>0$, there exists a $\delta=\delta(\varepsilon, \mathbf{T})>0$, such that for any $\left(0, \tilde{x}_{0}\right) \in[0, \mathbf{T}] \times \mathbf{R}$, and $\left|\widetilde{x}_{0}-x_{0}\right|<\delta$, then:

$$
\left|x\left(t ; 0, \widetilde{x}_{0}\right)-x\left(t ; 0, x_{0}\right)\right|<\varepsilon, t \in\left[0, t_{1}\right] \bigcup\left(t_{i}, s_{i}\right] \bigcup\left(s_{i}, t_{i+1}\right], i \in \mathbf{N}^{+}
$$

Definition 2. The solution $X\left(\cdot ; s_{i}, x_{s_{i}}\right) \in \mathbf{C}\left(\left[s_{i}, t_{i+1}\right], \mathbf{R}\right)$ of Equations (3) and (5) depends uniformly and continuously on the initial point $\left(s_{i}, x_{s_{i}}\right)$, if for any $\varepsilon>0, \mathbf{T}>0$, there exists a $\delta=\delta(\varepsilon, \mathbf{T})>0$, such that for any $\left(s_{i}, \widetilde{x}_{s_{i}}\right) \in[0, \mathbf{T}] \times \mathbf{R}$, and $\left|\widetilde{x}_{s_{i}}-x_{s_{i}}\right|<\delta$, then:

$$
\left|X\left(t ; s_{i}, \widetilde{x}_{s_{i}}\right)-X\left(t ; s_{i}, x_{s_{i}}\right)\right|<\varepsilon, t \in\left[s_{i}, t_{i+1}\right], i \in \mathbf{N}^{+} .
$$

We introduce the following assumptions for further discussion:

$\left[H_{1}\right]$ The function $\mathbf{f}: \mathbf{J} \times \mathbf{R} \rightarrow \mathbf{R}$ is continuous and $\mathbf{h}_{\mathbf{i}} \in \mathbf{C}\left(\left[t_{i}, s_{i}\right] \times \mathbf{R}, \mathbf{R}\right), i \in \mathbf{N}^{+}$.

$\left[H_{2}\right]$ There exists an $\mathbf{L}_{\mathbf{f}}>0$, such that $|\mathbf{f}(t, x)-\mathbf{f}(t, y)| \leq \mathbf{L}_{\mathbf{f}}|x-y|$, for each $t \in\left[s_{i}, t_{i+1}\right], i \in$ $\mathbf{N}$, and for all $x, y \in \mathbf{R}$.

$\left[H_{3}\right]$ There exists a positive constant $\mathbf{L}_{\mathbf{h}_{\mathbf{i}}}, i \in \mathbf{N}^{+}$, such that $\left|\mathbf{h}_{\mathbf{i}}(t, x)-\mathbf{h}_{\mathbf{i}}(t, y)\right| \leq \mathbf{L}_{\mathbf{h}_{\mathbf{i}}} \mid x-$ $y \mid$, for each $t \in\left[t_{i}, s_{i}\right], i \in \mathbf{N}^{+}$, and for all $x, y \in \mathbf{R}$.

$\left[H_{4}\right]$ The solutions of Equations (3) or (5) depend uniformly and continuously on the initial point.

$\left[H_{5}\right]$ The functions $\mathbf{h}_{\mathbf{i}}(t, x), i \in \mathbf{N}^{+}$, are uniformly bounded, i.e., for any $i \in \mathbf{N}^{+}$, there exists an $\mathbf{M}>0$, for any $x \in \mathbf{R}, t \in \mathbf{J}$, such that $\left|\mathbf{h}_{\mathbf{i}}(t, x)\right| \leq \mathbf{M}$.

Theorem 1. Assume that $\left[H_{1}\right]-\left[H_{3}\right]$ are satisfied. Then the solution of Equation (1) depends continuously on the initial point $\left(0, x_{0}\right)$ at the random impulse and junction points.

Proof. Let $\varepsilon$ and $\mathbf{T}$ be two arbitrary positive constants and $\Omega=\left\{t_{1}, s_{1}, t_{2}, s_{2}, \cdots\right\}$ be an arbitrary set of impulse and junction points. Note that if $t_{i} \rightarrow \infty$ as $i \rightarrow \infty$, then for any selection of the set of impulse points and junction points, there exists $k \in \mathbf{N}$ such that $s_{k}<\mathbf{T} \leq t_{k+1}$, i.e., there exists at most $k$ impulse points and at most $k$ junction points belonging to the interval $[0, \mathbf{T}]$. Without loss of generality, we assume that $\mathbf{T}=t_{k+1}$.

We divide the proof into several cases.

Case 1. For the interval $\left(s_{k}, t_{k+1}\right]$, the solutions of Equations (3) and (4) are given in Reference [14]:

$$
X\left(t ; s_{k}, x_{s_{k}}\right)=x_{s_{k}}+\int_{s_{k}}^{t} \mathbf{f}\left(s, X\left(s ; s_{k}, x_{s_{k}}\right)\right) d s,
$$

and:

$$
X\left(t ; s_{k}, \widetilde{x}_{s_{k}}\right)=\widetilde{x}_{s_{k}}+\int_{s_{k}}^{t} \mathbf{f}\left(s, \widetilde{X}\left(s ; s_{k}, x_{s_{k}}\right)\right) d s .
$$


Assume that there exists a $\delta_{k, k+1}=\delta_{k, k+1}(\varepsilon, \mathbf{T})$, where $0<\delta_{k, k+1}<\varepsilon$ and $\left|\widetilde{x}_{s_{k}}-x_{s_{k}}\right|<\delta_{k, k+1}$, linking Equations (8) and (9), we get:

$$
\begin{aligned}
& \left|X\left(t ; s_{k}, \widetilde{x}_{s_{k}}\right)-X\left(t ; s_{k}, x_{s_{k}}\right)\right| \\
\leq & \left|\widetilde{x}_{s_{k}}-x_{s_{k}}\right|+\mathbf{L}_{\mathbf{f}} \int_{s_{k}}^{t}\left|X\left(s ; s_{k}, \widetilde{x}_{s_{k}}\right)-X\left(s ; s_{k}, x_{s_{k}}\right)\right| d s \\
\leq & \delta_{k, k+1}+\mathbf{L}_{\mathbf{f}} \int_{s_{k}}^{t}\left|X\left(s ; s_{k}, \widetilde{x}_{s_{k}}\right)-X\left(s ; s_{k}, x_{s_{k}}\right)\right| d s .
\end{aligned}
$$

From Reference [36] (Theorem 1.1, therein), we get:

$$
\left|X\left(t ; s_{k}, \widetilde{x}_{s_{k}}\right)-X\left(t ; s_{k}, x_{s_{k}}\right)\right| \leq \delta_{k, k+1} e^{\mathbf{L}_{\mathbf{f}}\left(t_{k+1}-s_{k}\right)} .
$$

Since $0<\delta_{k, k+1}<\varepsilon$, we have:

$$
\left|X\left(t ; s_{k}, \widetilde{x}_{s_{k}}\right)-X\left(t ; s_{k}, x_{s_{k}}\right)\right|<\varepsilon, t \in\left(s_{k}, t_{k+1}\right] .
$$

Case 2. For the interval $\left(t_{k}, s_{k}\right]$, we have the following expression of the solutions, respectively:

$$
X\left(t ; t_{k}, x\left(t_{k}^{+}\right)\right)=\mathbf{h}_{\mathbf{k}}\left(t, x\left(t_{k}^{-}\right)\right) \text {and } X\left(t ; t_{k}, \widetilde{x}\left(t_{k}\right)\right)=\mathbf{h}_{\mathbf{k}}\left(t, \widetilde{x}\left(t_{k}^{-}\right)\right) .
$$

Assume there exists $\delta_{k, k}<\delta_{k, k+1}$, where $\left|\widetilde{x}\left(t_{k}^{+}\right)-x\left(t_{k}^{+}\right)\right|<\delta_{k k}$, then we have:

$$
\left|X\left(t ; t_{k}, \widetilde{x}\left(t_{k}^{+}\right)\right)-X\left(t ; t_{k}, x\left(t_{k}^{+}\right)\right)\right| \leq \mathbf{L}_{\mathbf{h}_{\mathbf{k}}}\left|\widetilde{x}\left(t_{k}^{-}\right)-x\left(t_{k}^{-}\right)\right| .
$$

For $\left(s_{k-1}, t_{k}\right]$, similar to Equation (10), we assume there exists a $\delta_{k-1, k}<\frac{\delta_{k, k}}{\mathbf{L}_{\mathbf{h}_{\mathbf{k}}} e^{\mathbf{L}_{\mathbf{f}}\left(t_{k}-s_{k-1}\right)}}$. Then we obtain:

$$
\left|X\left(t ; s_{k-1}, \widetilde{x}_{s_{k-1}}\right)-X\left(t ; s_{k-1}, x_{s_{k-1}}\right)\right| \leq \delta_{k-1, k-1} e^{\mathbf{L}_{\mathbf{f}}\left(t_{k}-s_{k-1}\right)}<\frac{\delta_{k, k}}{\mathbf{L}_{\mathbf{h}_{\mathbf{k}}}}<\varepsilon, t \in\left(s_{k-1}, t_{k}\right] .
$$

For $t=t_{k}$, we get:

$$
\left|X\left(t_{k} ; s_{k-1}, \tilde{x}_{s_{k-2}}\right)-X\left(t_{k} ; s_{k-1}, x_{s_{k-1}}\right)\right|<\frac{\delta_{k, k}}{\mathbf{L}_{\mathbf{h}_{\mathbf{k}}}}
$$

and:

$$
\left|\mathbf{h}_{\mathbf{k}}\left(t_{k}, \widetilde{x}\left(t_{k}^{-}\right)\right)-\mathbf{h}_{\mathbf{k}}\left(t_{k}, x\left(t_{k}^{-}\right)\right)\right|<\mathbf{L}_{\mathbf{h}_{\mathbf{k}}} \frac{\delta_{k, k}}{\mathbf{L}_{\mathbf{h}_{\mathbf{k}}}}<\delta_{k, k} .
$$

Therefore, Equation (11) becomes:

$$
\left|X\left(t ; t_{k}, \widetilde{x}\left(t_{k}^{+}\right)\right)-X\left(t ; t_{k}, x\left(t_{k}^{+}\right)\right)\right|<\delta_{k, k}<\delta_{k, k+1}<\varepsilon, t \in\left(t_{k}, s_{k}\right] .
$$

From the procedure of Equations (12) and (13), we finally establish the following facts:

For the interval $\left(t_{1}, s_{1}\right]$, if there is a $\delta_{11}<\delta_{12}$, where $\left|\widetilde{x}\left(t_{1}^{+}\right)-x\left(t_{1}^{+}\right)\right|<\delta_{11}$, then:

$$
\left|X\left(t ; t_{1}, \widetilde{x}\left(t_{1}^{+}\right)\right)-X\left(t ; t_{1}, x\left(t_{1}^{+}\right)\right)\right| \leq \mathbf{L}_{\mathbf{h}_{1}}\left|\widetilde{x}\left(t_{1}^{-}\right)-x\left(t_{1}^{-}\right)\right| .
$$

For the interval $\left[0, t_{1}\right]$, we assume there is a $\delta_{01}<\frac{\delta_{11}}{\mathbf{L}_{\mathbf{h}_{1}} e^{\mathrm{L}_{\mathrm{f}} t_{1}}}$, where $\left|\widetilde{x}_{0}-x_{0}\right|<\delta_{01}$. Thus, we obtain:

$$
\left|X\left(t ; 0, \widetilde{x}_{0}\right)-X\left(t ; 0, x_{0}\right)\right| \leq \delta_{01} e^{\mathbf{L}_{\mathbf{f}} t_{1}}<\frac{\delta_{11}}{\mathbf{L}_{\mathbf{h}_{1}}}<\varepsilon, \quad t \in\left[0, t_{1}\right] .
$$

For $t=t_{1}$, we get:

$$
\left|X\left(t_{1} ; 0, \widetilde{x}_{0}\right)-X\left(t_{1} ; 0, x_{0}\right)\right|<\frac{\delta_{11}}{\mathbf{L}_{\mathbf{h}_{1}}}
$$


and hence, we have:

$$
\left|h_{1}\left(t_{1}, x\left(t_{1}^{-}\right)\right)-h_{1}\left(t_{1}, x\left(t_{1}^{-}\right)\right)\right|<\mathbf{L}_{\mathbf{h}_{1}} \frac{\delta_{11}}{\mathbf{L}_{\mathbf{h}_{\mathbf{1}}}}<\delta_{11} .
$$

Thus, Equation (14) becomes:

$$
\left|X\left(t ; t_{1}, \widetilde{x}\left(t_{1}^{+}\right)\right)-X\left(t ; t_{1}, x\left(t_{1}^{+}\right)\right)\right|<\delta_{11}<\delta_{12}<\varepsilon, \quad t \in\left(t_{1}, s_{1}\right] .
$$

Next, we notice that:

$$
\begin{aligned}
\delta_{01}= & \delta_{01}\left(\varepsilon, \mathbf{T}, \delta_{11}\right), \\
& \vdots \\
\delta_{k-1, k}= & \delta_{k-1, k}\left(\varepsilon, \mathbf{T}, \delta_{k k}\right), \\
\delta_{k k}= & \delta_{k k}\left(\varepsilon, \mathbf{T}, \delta_{k, k+1}\right), \\
\delta_{k, k+1}= & \delta_{k, k+1}(\varepsilon, \mathbf{T}) .
\end{aligned}
$$

Therefore, we have $\delta_{01}=\delta_{01}(\varepsilon, \mathbf{T})$. Considering Equations (7) and (15), we obtain, for any $\varepsilon>0$, there exists a $\delta_{01}>0$, where $\left|\widetilde{x}_{0}-x_{0}\right|<\delta_{01}$, thus:

$$
\left|x\left(t ; 0, \widetilde{x}_{0}\right)-x\left(t ; 0, x_{0}\right)\right|<\varepsilon, t \in\left[0, t_{1}\right],
$$

and from Equation (16) we get:

$$
\left|\mathbf{h}_{\mathbf{1}}\left(t_{1}, \widetilde{x}\left(t_{1}^{-}\right)\right)-\mathbf{h}_{\mathbf{1}}\left(t_{1}, x\left(t_{1}^{-}\right)\right)\right|=\left|\widetilde{x}\left(t_{1}^{+}\right)-x\left(t_{1}^{+}\right)\right|<\delta_{11} .
$$

Taking into account Equations (17) and (7), we find that:

$$
\left|x\left(t ; 0, \widetilde{x}_{0}\right)-x\left(t ; 0, x_{0}\right)\right|<\varepsilon, t \in\left(t_{1}, s_{1}\right],
$$

and:

$$
\left|\mathbf{h}_{\mathbf{1}}\left(s_{1}, \widetilde{x}\left(t_{1}^{-}\right)\right)-\mathbf{h}_{\mathbf{1}}\left(s_{1}, x\left(t_{1}^{-}\right)\right)\right|=\left|\widetilde{x}_{s_{1}}-x_{s_{1}}\right|<\delta_{12} .
$$

According to Equation (7) again, we obtain:

$$
\left|x\left(t ; 0, \widetilde{x}_{0}\right)-x\left(t ; 0, x_{0}\right)\right|<\varepsilon, t \in\left(s_{1}, t_{2}\right] .
$$

Similarly, we achieve the following conclusion:

$$
\left|x\left(t ; 0, \widetilde{x}_{0}\right)-x\left(t ; 0, x_{0}\right)\right|<\varepsilon, t \in\left(t_{k}, s_{k}\right],
$$

and:

$$
\left|\mathbf{h}_{\mathbf{k}}\left(s_{k}, \widetilde{x}\left(t_{k}^{-}\right)\right)-\mathbf{h}_{\mathbf{k}}\left(s_{k}, x\left(t_{k}^{-}\right)\right)\right|=\left|\widetilde{x}_{s_{k}}-x_{s_{k}}\right|<\delta_{k, k+1} .
$$

Then, it is further determined:

$$
\left|x\left(t ; 0, \widetilde{x}_{0}\right)-x\left(t ; 0, x_{0}\right)\right|<\varepsilon, t \in\left(s_{k}, t_{k+1}\right] .
$$

By Equations (18)-(21), we get that for any $\varepsilon>0$ and $\widetilde{x}_{0}, x_{0} \in \mathbf{R}$, there exists a $\delta>0$, where $\mid \widetilde{x}_{0}-$ $x_{0} \mid<\delta$, such that $\left|x\left(t ; 0, \widetilde{x}_{0}\right)-x\left(t ; 0, x_{0}\right)\right|<\varepsilon$ for $t \in\left[0, t_{1}\right] \cup\left(t_{i}, s_{i}\right] \cup\left(s_{i}, t_{i+1}\right], i=1,2, \cdots, k$, where $\delta=$ $\delta_{01}(\varepsilon, \mathbf{T})$. By Definition 1 , the proof is completed. 
Theorem 2. Suppose that $\left[H_{1}\right]-\left[H_{3}\right]$ are satisfied. Then the solution of Equation (2) depends continuously on the initial point $\left(0, x_{0}\right)$ for random impulse and junction points.

Proof. Let $\varepsilon$ and $\mathbf{T}$ be two arbitrary positive constants like in Theorem 1. We divide the proof into two cases.

Case 1. For the interval $\left(s_{k}, t_{k+1}\right]$, the representation of the solutions of Equations (5) and (6) are given by [14]:

$$
X\left(t ; s_{k}, x_{s_{k}}\right)=x_{s_{k}}+\frac{1}{\Gamma(\alpha)} \int_{s_{k}}^{t}(t-s)^{\alpha-1} \mathbf{f}\left(s, X\left(s ; s_{k}, x_{s_{k}}\right)\right) d s
$$

and:

$$
X\left(t ; s_{k}, \widetilde{x}_{s_{k}}\right)=\widetilde{x}_{s_{k}}+\frac{1}{\Gamma(\alpha)} \int_{s_{k}}^{t}(t-s)^{\alpha-1} \mathbf{f}\left(s, X\left(s ; s_{k}, \widetilde{x}_{s_{k}}\right)\right) d s .
$$

Assume that there exists a $\delta_{k, k+1}=\delta_{k, k+1}(\varepsilon, \mathbf{T}), 0<\delta_{k, k+1}<\varepsilon$, where $\left|\widetilde{x}_{s_{k}}-x_{s_{k}}\right|<\delta_{k, k+1}$, then from Equations (22) and (23), we have:

$$
\begin{aligned}
& \left|X\left(t ; s_{k}, \widetilde{x}_{s_{k}}\right)-X\left(t ; s_{k}, x_{s_{k}}\right)\right| \\
\leq & \left|\widetilde{x}_{s_{k}}-x_{s_{k}}\right|+\frac{\mathbf{L}_{\mathbf{f}}}{\Gamma(\alpha)} \int_{s_{k}}^{t}(t-s)^{\alpha-1}\left|X\left(s ; s_{k}, \widetilde{x}_{s_{k}}\right)-X\left(s ; s_{k}, x_{s_{k}}\right)\right| d s \\
\leq & \delta_{k, k+1}+\frac{\mathbf{L}_{\mathbf{f}}}{\boldsymbol{\Gamma}(\alpha)} \int_{s_{k}}^{t}(t-s)^{\alpha-1}\left|X\left(s ; s_{k}, \widetilde{x}_{s_{k}}\right)-X\left(s ; s_{k}, x_{s_{k}}\right)\right| d s .
\end{aligned}
$$

Using Reference [37] (Corollary 2, therein), we get:

$$
\left|X\left(t ; s_{k}, \widetilde{x}_{s_{k}}\right)-X\left(t ; s_{k}, x_{s_{k}}\right)\right| \leq \delta_{k, k+1} \mathbf{E}_{\alpha}\left(\mathbf{L}_{\mathbf{f}}\left(t_{k+1}-s_{k}\right)^{\alpha}\right),
$$

where $\mathbf{E}_{\alpha}$ is the standard Mittag-Leffler function [35] defined as:

$$
\mathbf{E}_{\alpha}(z)=\sum_{k=0}^{\infty} \frac{z^{k}}{\Gamma(k \alpha+1)}, \quad z \in \mathbb{C}
$$

Owing to $0<\delta_{k, k+1}<\varepsilon$, one can get:

$$
\left|X\left(t ; s_{k}, \widetilde{x}_{s_{k}}\right)-X\left(t ; s_{k}, x_{s_{k}}\right)\right|<\varepsilon, t \in\left(s_{k}, t_{k+1}\right] .
$$

Case 2. From the interval $\left(t_{k}, s_{k}\right]$, the expression of the solutions are given by:

$$
X\left(t ; t_{k}, x\left(t_{k}^{+}\right)\right)=\mathbf{h}_{\mathbf{k}}\left(t, x\left(t_{k}^{-}\right)\right) \text {and } X\left(t ; t_{k}, \widetilde{x}\left(t_{k}\right)\right)=\mathbf{h}_{\mathbf{k}}\left(t, \widetilde{x}\left(t_{k}^{-}\right)\right) .
$$

Assume that there exists a $\delta_{k k}<\delta_{k, k+1}$, where $\left|\widetilde{x}\left(t_{k}^{+}\right)-x\left(t_{k}^{+}\right)\right|<\delta_{k k}$, then we have:

$$
\left|X\left(t ; t_{k}, \widetilde{x}\left(t_{k}^{+}\right)\right)-X\left(t ; t_{k}, x\left(t_{k}^{+}\right)\right)\right| \leq \mathbf{L}_{\mathbf{h}_{\mathbf{k}}}\left|\widetilde{x}\left(t_{k}^{-}\right)-x\left(t_{k}^{-}\right)\right| .
$$

Similar to the above procedure, for the interval $\left(s_{k-1}, t_{k}\right]$, one has:

$$
\left|X\left(t ; s_{k-1}, \widetilde{x}_{s_{k-1}}\right)-X\left(t ; s_{k-1}, x_{s_{k-1}}\right)\right| \leq \delta_{k-1, k} \mathbf{E}_{\alpha}\left(L_{f}\left(t_{k}-s_{k-1}\right)^{\alpha}\right) .
$$

Since $\delta_{k-1, k}<\frac{\delta_{k k}}{\mathbf{L}_{\mathbf{h}_{\mathbf{k}}} \mathbf{E}_{\alpha}\left(\mathbf{L}_{\mathbf{f}}\left(t_{k}-s_{k-1}\right)^{\alpha}\right)}$, one can obtain:

$$
\left|X\left(t ; s_{k-1}, \widetilde{x}_{s_{k-1}}\right)-X\left(t ; s_{k-1}, x_{s_{k-1}}\right)\right|<\frac{\delta_{k k}}{\mathbf{L}_{\mathbf{h}_{\mathbf{k}}}}<\varepsilon, t \in\left(s_{k-1}, t_{k}\right] .
$$


For $t=t_{k}$, we get:

$$
\left|X\left(t_{k} ; s_{k-1}, \widetilde{x}_{s_{k-1}}\right)-X\left(t_{k} ; s_{k-1}, x_{s_{k-1}}\right)\right|<\frac{\delta_{k k}}{\mathbf{L}_{\mathbf{h}_{\mathbf{k}}}}
$$

and then:

$$
\left|g_{k}\left(t_{k}, x\left(t_{k}^{-}\right)\right)-g_{k}\left(t_{k}, x\left(t_{k}^{-}\right)\right)\right|<\mathbf{L}_{\mathbf{h}_{\mathbf{k}}} \frac{\delta_{k k}}{\mathbf{L}_{\mathbf{h}_{\mathbf{k}}}}<\delta_{k k} .
$$

Therefore, (24) becomes:

$$
\left|X\left(t ; t_{k}, \widetilde{x}\left(t_{k}^{+}\right)\right)-X\left(t ; t_{k}, x\left(t_{k}^{+}\right)\right)\right|<\delta_{k k}<\varepsilon
$$

From the above, one can deduce that for the interval $\left(t_{1}, s_{1}\right]$, there exists a $\delta_{11}<\delta_{12}$, and if $\left|\widetilde{x}\left(t_{1}^{-}\right)-x\left(t_{1}^{-}\right)\right|<\delta_{11}$, then:

$$
\left|X\left(t ; t_{1}, x\left(t_{1}^{+}\right)\right)-X\left(t ; t_{1}, x\left(t_{1}^{+}\right)\right)\right| \leq \mathbf{L}_{\mathbf{h}_{\mathbf{1}}}\left|\widetilde{x}\left(t_{1}^{-}\right)-x\left(t_{1}^{-}\right)\right| .
$$

For $\left[0, t_{1}\right]$, there is $\delta_{01}<\frac{\delta_{11}}{\mathbf{L}_{\mathbf{h}_{1}} \mathbf{E}_{\alpha}\left(\mathbf{L}_{\mathbf{f}} t_{1}^{\alpha}\right)}$, if $\left|\widetilde{x}_{0}-x_{0}\right|<\delta_{01}$, then:

$$
\left|X\left(t ; 0, \widetilde{x}_{0}\right)-X\left(t ; 0, x_{0}\right)\right| \leq \delta_{01} \mathbf{E}_{\alpha}\left(\mathbf{L}_{\mathbf{f}} t_{1}^{\alpha}\right)<\frac{\delta_{11}}{\mathbf{L}_{\mathbf{h}_{1}}}<\varepsilon, t \in\left[0, t_{1}\right] .
$$

Thus, for $t=t_{1}$, we have:

$$
\left|X\left(t_{1} ; 0, \widetilde{x}_{0}\right)-X\left(t_{1} ; 0, x_{0}\right)\right|<\frac{\delta_{11}}{\mathbf{L}_{\mathbf{h}_{1}}}
$$

and then:

$$
\left|\mathbf{h}_{\mathbf{1}}\left(t_{1}, \widetilde{x}\left(t_{1}\right)\right)-\mathbf{h}_{\mathbf{1}}\left(t_{1}, x\left(t_{1}\right)\right)\right|<\delta_{11}
$$

and:

$$
\left|X\left(t ; t_{1}, x\left(t_{1}^{+}\right)\right)-X\left(t ; t_{1}, x\left(t_{1}^{+}\right)\right)\right|<\delta_{11}<\delta_{12}<\varepsilon, t \in\left(t_{1}, s_{1}\right] .
$$

Similar to Theorem 1 , we reach the conclusion.

Theorem 3. Assume that $\left[H_{1}\right],\left[H_{4}\right]$, and $\left[H_{5}\right]$ are satisfied. The solution of Equation (1) depends continuously on the initial point $\left(0, x_{0}\right)$ at the random impulse and junction points provided that $2 \mathbf{M} \leq\left|\widetilde{x}_{s_{i}}-x_{s_{i}}\right|$.

Proof. We divide proof into several steps.

Step 1. According to $\left[H_{4}\right]$, assume there is a $\delta_{k, k+1}=\delta_{k, k+1}(\varepsilon, \mathbf{T})$, where $0<\delta_{k, k+1}<\varepsilon$, if $\left|\widetilde{x}_{s_{k}}-x_{s_{k}}\right|<\delta_{k, k+1}$, then:

$$
\left|X\left(t ; s_{k}, \widetilde{x}_{s_{k}}\right)-X\left(t ; s_{k}, x_{s_{k}}\right)\right|<\varepsilon, t \in\left(s_{k}, t_{k+1}\right] .
$$

Step 2. For the interval $\left(t_{k}, s_{k}\right]$, according to $\left[H_{5}\right]$, we obtain:

$$
\begin{aligned}
\left|X\left(t ; t_{k}, \widetilde{x}\left(t_{k}^{+}\right)\right)-X\left(t ; t_{k}, x\left(t_{k}^{+}\right)\right)\right| & =\left|\mathbf{h}_{\mathbf{k}}\left(t, \widetilde{x}\left(t_{k}^{-}\right)\right)-\mathbf{h}_{\mathbf{k}}\left(t, x\left(t_{k}^{-}\right)\right)\right| \\
& \leq 2 \mathbf{M} \leq\left|\widetilde{x}_{s_{k}}-x_{s_{k}}\right|<\delta_{k, k+1}<\varepsilon, t \in\left(t_{k}, s_{k}\right] .
\end{aligned}
$$

For $t=s_{k}$, we get:

$$
\left|\mathbf{h}_{\mathbf{k}}\left(s_{k}, \widetilde{x}\left(t_{k}^{-}\right)\right)-\mathbf{h}_{\mathbf{k}}\left(s_{k}, x\left(t_{k}^{-}\right)\right)\right|<\delta_{k, k+1} .
$$

Step 3. In this step, we check the continuity of the solutions in the interval $\left(s_{k-1}, t_{k}\right]$. From Equation (25), we assume there exists a $\delta_{k-1, k}$ depending on $\varepsilon, \mathbf{T}, \delta_{k, k+1}$. For brevity, we denote 
$\delta_{k-1, k}=\delta_{k-1, k}\left(\varepsilon, \mathbf{T}, \delta_{k, k+1}\right)$, where $0<\delta_{k-1, k}<\varepsilon$. If $\left|\widetilde{x}_{s_{k-1}}-x_{s_{k-1}}\right|<\delta_{k-1, k}$, then by $\left[H_{4}\right]$ we deduce that:

$$
\left|X\left(t ; s_{k-1}, \widetilde{x}_{s_{k-1}}\right)-X\left(t ; s_{k-1}, x_{s_{k-1}}\right)\right|<\varepsilon, t \in\left(s_{k-1}, t_{k}\right] .
$$

Step 4. For $\left(t_{k-1}, s_{k-1}\right]$, we get:

$$
\begin{aligned}
\left|X\left(t ; t_{k-1}, \widetilde{x}\left(t_{k-1}^{+}\right)\right)-X\left(t ; t_{k-1}, x\left(t_{k-1}^{+}\right)\right)\right| & =\left|\mathbf{h}_{\mathbf{k}-\mathbf{1}}\left(t, \widetilde{x}\left(t_{k-1}^{-}\right)\right)-\mathbf{h}_{\mathbf{k}-\mathbf{1}}\left(t, x\left(t_{k-1}^{-}\right)\right)\right| \\
& \leq \mathbf{M} \leq\left|\widetilde{x}_{s_{k-1}}-x_{s_{k-1}}\right|<\delta_{k-1, k}<\varepsilon, t \in\left(t_{k-1}, s_{k-1}\right] .
\end{aligned}
$$

For $t=s_{k-1}$, we have:

$$
\left|\mathbf{h}_{\mathbf{k}-\mathbf{1}}\left(s_{k-1}, \widetilde{x}\left(t_{k-1}^{-}\right)\right)-\mathbf{h}_{\mathbf{k}-\mathbf{1}}\left(s_{k-1}, x\left(t_{k-1}^{-}\right)\right)\right|<\delta_{k-1, k} .
$$

Similar to the above steps, we have the following general results.

Step $2 k$. For the interval $\left(t_{1}, s_{1}\right]$, it follows that:

$$
\left|X\left(t ; t_{1}, \widetilde{x}\left(t_{1}^{+}\right)\right)-X\left(t ; t_{1}, x\left(t_{1}^{+}\right)\right)\right|=\left|\mathbf{h}_{\mathbf{1}}\left(t, \widetilde{x}\left(t_{1}^{-}\right)\right)-\mathbf{h}_{\mathbf{1}}\left(t, x\left(t_{1}^{-}\right)\right)\right|<\delta_{12}<\varepsilon, t \in\left(t_{1}, s_{1}\right] .
$$

For $t=s_{1}$, we have:

$$
\left|\mathbf{h}_{\mathbf{1}}\left(s_{1}, \widetilde{x}\left(t_{1}^{-}\right)\right)-\mathbf{h}_{\mathbf{1}}\left(s_{1}, x\left(t_{1}^{-}\right)\right)\right|<\delta_{12} .
$$

Step $2 k+1$. For $\left[0, t_{1}\right]$, assume there exists a $\delta_{01}=\delta_{01}\left(\varepsilon, \mathbf{T}, \delta_{12}\right)$, where $0<\delta_{01}<\varepsilon$, if $\left|\widetilde{x}_{0}-x_{0}\right|<\varepsilon$, then:

$$
\left|X\left(t ; 0, \widetilde{x}_{0}\right)-X\left(t ; 0, x_{0}\right)\right|<\varepsilon, t \in\left[0, t_{1}\right] .
$$

Finally, we notice that:

$$
\begin{aligned}
\delta_{01}= & \delta_{01}\left(\varepsilon, \mathbf{T}, \delta_{12}\right), \\
& \vdots \\
\delta_{k-1, k}= & \delta_{k-1, k}\left(\varepsilon, \mathbf{T}, \delta_{k, k+1}\right), \\
\delta_{k, k+1}= & \delta_{k, k+1}(\varepsilon, \mathbf{T}) .
\end{aligned}
$$

Therefore, we have $\delta_{01}=\delta_{01}(\varepsilon, \mathbf{T})$.

Now we apply the above results to the impulse case.

Step $1^{\prime}$. Based on Equation (7) and Step $2 k+1$, we obtain for any $\varepsilon>0$, there exists a $\delta_{01}>$ 0 , such that if $\left|\widetilde{x}_{0}-x_{0}\right|<\delta_{01}$, then:

$$
\left|x\left(t ; 0, \widetilde{x}_{0}\right)-x\left(t ; 0, x_{0}\right)\right|<\varepsilon, t \in\left[0, t_{1}\right] .
$$

Step $2^{\prime}$. Using Equation (7) and taking Step 2k into account, we find that:

$$
\left|x\left(t ; 0, \widetilde{x}_{0}\right)-x\left(t ; 0, x_{0}\right)\right|<\varepsilon, t \in\left(t_{1}, s_{1}\right] .
$$

From Equation (26), we have:

$$
\left|\mathbf{h}_{\mathbf{1}}\left(s_{1}, \widetilde{x}\left(t_{1}^{-}\right)\right)-\mathbf{h}_{\mathbf{1}}\left(s_{1}, x\left(t_{1}^{-}\right)\right)\right|=\left|\widetilde{x}_{s_{1}}-x_{s_{1}}\right|<\delta_{12} .
$$

Step $3^{\prime}$. According to Equation (7) and Step 2k - 1, it follows that:

$$
\left|x\left(t ; 0, \widetilde{x}_{0}\right)-x\left(t ; 0, x_{0}\right)\right|<\varepsilon, t \in\left(s_{1}, t_{2}\right] .
$$


Step $4^{\prime}$. Again by Equation (7) and considering Step $2 \mathrm{k}-2$, we have:

$$
\left|x\left(t ; 0, \widetilde{x}_{0}\right)-x\left(t ; 0, x_{0}\right)\right|<\varepsilon, t \in\left(t_{2}, s_{2}\right],
$$

and:

$$
\left|\mathbf{h}_{\mathbf{2}}\left(s_{2}, \widetilde{x}\left(t_{2}^{-}\right)\right)-\mathbf{h}_{\mathbf{2}}\left(s_{2}, x\left(t_{2}^{-}\right)\right)\right|=\left|\widetilde{x}_{s_{2}}-x_{s_{2}}\right|<\delta_{23} .
$$

Similarly, we arrive at:

Step $(2 k)^{\prime} .\left|x\left(t ; 0, \widetilde{x}_{0}\right)-x\left(t ; 0, x_{0}\right)\right|<\varepsilon, t \in\left(t_{k}, s_{k}\right]$.

Thus, from Equation (25), we get:

$$
\left|\mathbf{h}_{\mathbf{k}}\left(s_{k}, \widetilde{x}\left(t_{k}^{-}\right)\right)-\mathbf{h}_{\mathbf{k}}\left(s_{k}, x\left(t_{k}^{-}\right)\right)\right|=\left|\widetilde{x}_{s_{k}}-x_{s_{k}}\right|<\delta_{k, k+1} .
$$

Step $(2 k+1)^{\prime} .\left|x\left(t ; 0, \widetilde{x}_{0}\right)-x\left(t ; 0, x_{0}\right)\right|<\varepsilon, t \in\left(s_{k}, t_{k+1}\right]$.

From Step $1^{\prime}$ to Step $(2 k+1)^{\prime}$, we get that for any $\varepsilon>0, \widetilde{x}_{0}, x_{0} \in \mathbf{R}$, there exists a $\delta>$ 0 , such that if $\left|\widetilde{x}_{0}-x_{0}\right|<\delta$, then $\left|x\left(t ; 0, \widetilde{x}_{0}\right)-x\left(t ; 0, x_{0}\right)\right|<\varepsilon$, for $t \in\left[0, t_{1}\right] \cup\left(t_{i}, s_{i}\right] \cup\left(s_{i}, t_{i+1}\right], i=$ $1,2, \cdots, k$, and $\delta=\delta_{01}(\varepsilon, \mathbf{T})$.

By repeating the same proof procedure in Theorem 3, we have the result:

Remark 1. Assume that $\left[\mathrm{H}_{1}\right],\left[\mathrm{H}_{4}\right]$, and $\left[\mathrm{H}_{5}\right]$ are satisfied. Then the solution of Equation (2) depends continuously on the initial point $\left(0, x_{0}\right)$ at the random impulse and junction points, provided that $2 \mathbf{M} \leq$ $\left|\widetilde{x}_{s_{i}}-x_{s_{i}}\right|$.

\section{Numerical Examples}

Let $\varepsilon$ and $\mathbf{T}$ be two arbitrary positive constants.

Example 1. Consider:

$$
\left\{\begin{array}{l}
x^{\prime}(t)=a x(t), t \in\left(s_{i}, t_{i+1}\right], i \in \mathbf{N}, a>0, \\
x\left(t_{i}^{+}\right)=\frac{\varepsilon}{2 e^{a t}} \cos \sqrt{t_{i} \mid x\left(t_{i}^{-}\right)} \mid, i \in \mathbf{N}^{+}, \\
x(t)=\frac{\varepsilon}{2 e^{a t}} \cos \sqrt{t \mid x\left(t_{i}^{-}\right)} \mid, t \in\left(t_{i}, s_{i}\right], i \in \mathbf{N}^{+}, \\
x(0)=x_{0} .
\end{array}\right.
$$

Then the solution of Equation (27) can be analytically determined, namely:

$$
x(t)=\left\{\begin{array}{l}
e^{a t} x_{0}, \text { for } t \in\left(s_{0}, t_{1}\right], \\
\frac{\varepsilon}{2 e^{a t}} \cos \sqrt{t\left|x\left(t_{1}^{-}\right)\right|}, \text {for } t \in\left(t_{1}, s_{1}\right], \\
\frac{\varepsilon}{2 e^{a s_{1}}} \cos \sqrt{s_{1}\left|x\left(t_{1}^{-}\right)\right|} e^{a\left(t-s_{1}\right)}, \text { for } t \in\left(s_{1}, t_{2}\right], \\
\vdots \\
\frac{\varepsilon}{2 e^{a t}} \cos \sqrt{t\left|x\left(t_{m-1}^{-}\right)\right|}, \text {for } t \in\left(t_{m-1}, s_{m}\right], \\
\frac{\varepsilon}{2 e^{a s_{m}}}\left(\cos \sqrt{s_{m}\left|x\left(t_{m-1}^{-}\right)\right|} e^{a\left(t-s_{m}\right)}, \text { for } t \in\left(s_{m}, t_{m+1}\right],\right. \\
\vdots
\end{array}\right.
$$

Let $\mathbf{f}(t, x)=$ ax and $\mathbf{h}_{\mathbf{i}}(t, x)=\cos \sqrt{t|x|}$. Note that $\mathbf{h}_{\mathbf{i}} \in \mathbf{C}\left(\left[t_{i}, s_{i}\right] \times \mathbf{R}, \mathbf{R}\right), i \in \mathbf{N}^{+}$. For any $x, y \in \mathbf{R}$, $|\mathbf{f}(t, x)-\mathbf{f}(t, y)| \leq a|x-y|$ and $\left|\mathbf{h}_{\mathbf{i}}(t, x)-\mathbf{h}_{\mathbf{i}}(t, y)\right| \leq \frac{\varepsilon}{2 e a}|x-y|$. Set $\mathbf{L}_{\mathbf{f}}=a$ and $\mathbf{L}_{\mathbf{h}_{\mathbf{i}}}=\frac{\varepsilon}{2 e a}$. So, $\left[H_{1}\right]-\left[H_{3}\right]$ all hold. Therefore, all the assumptions in Theorem 1 are satisfied.

From Definition 2, choosing $\delta=\frac{\varepsilon}{e^{a \mathrm{~T}}}$, we find that the solution of Equation (27) without impulses satisfies uniform and continuous dependence on the initial point. Choosing $2 \mathbf{M} \leq \frac{\varepsilon}{e^{a \mathbf{T}}}$, then $\mathbf{h}_{i}(t, x)$ are uniformly bounded. Thus, the conditions $\left[H_{1}\right],\left[H_{4}\right]$, and $\left[H_{5}\right]$ hold, and all the assumptions of Theorem 3 are satisfied. 
The solutions of Equation (27) and the corresponding perturbation problem (with $x_{0}=1, \widetilde{x_{0}}=1.3, \mathbf{T}=$ $1, a=\frac{1}{2}, \varepsilon=\frac{1}{2}$ ) are shown in Figure 1 .

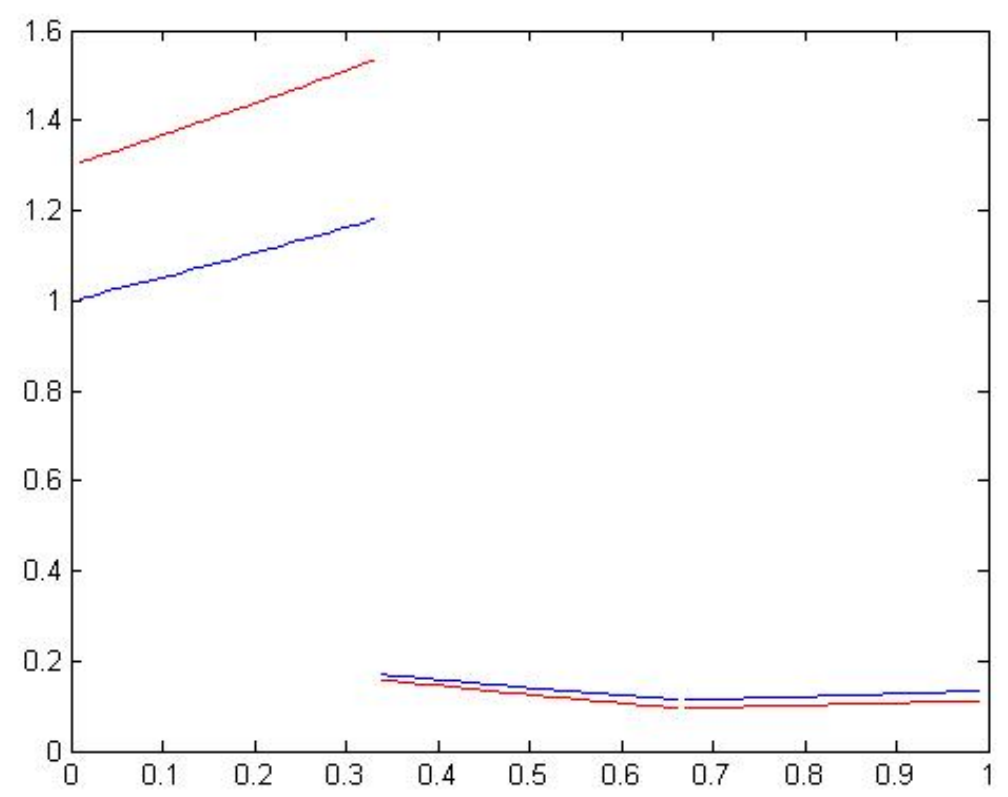

Figure 1. The blue line denotes the solution of Equation (27) and the red line denotes the corresponding perturbation problem.

Example 2. Consider:

$$
\left\{\begin{array}{l}
{ }^{C} \mathbf{D}_{s_{i}, t}^{\frac{1}{2}} x(t)=a x(t), t \in\left(s_{i}, t_{i+1}\right], i \in \mathbf{N}, a>0, \\
x\left(t_{i}^{+}\right)=\frac{\varepsilon}{2 \mathbf{E}_{\frac{1}{2}}\left(a t^{\frac{1}{2}}\right)}\left(\cos \sqrt{t_{i}\left|x\left(t_{i}^{-}\right)\right|}\right), i \in \mathbf{N}^{+}, \\
x(t)=\frac{\frac{\varepsilon}{\varepsilon}}{2 \mathbf{E}_{\frac{1}{2}}\left(a t^{\frac{1}{2}}\right)}\left(\cos \sqrt{t\left|x\left(t_{i}\right)^{-}\right|}\right), t \in\left(t_{i}, s_{i}\right], i \in \mathbf{N}^{+}, \\
x(0)=x_{0} .
\end{array}\right.
$$

Like Equation (27), let $\mathbf{f}(t, x)=a x$, and $\mathbf{h}_{\mathbf{i}}(t, x)=\frac{\varepsilon}{2 \mathbf{E}_{\frac{1}{2}}\left(a t^{\frac{1}{2}}\right)}(\cos \sqrt{t|x|})$. Choosing $\mathbf{L}_{\mathbf{f}}=a, \mathbf{L}_{\mathbf{h}_{\mathbf{i}}}=\frac{\varepsilon}{2 a^{2}}$, therefore, $\left[\mathrm{H}_{1}\right]-\left[\mathrm{H}_{3}\right]$ hold. Then, one can obtain the solution of Equation (29), namely:

$$
x(t)=\left\{\begin{array}{l}
x_{0} \mathbf{E}_{\frac{1}{2}}\left(a t^{\frac{1}{2}}\right), \text { for } t \in\left(s_{0}, t_{1}\right], \\
\frac{\varepsilon}{2 \mathbf{E}_{\frac{1}{2}}\left(a t^{\frac{1}{2}}\right)} \cos \sqrt{t\left|x\left(t_{1}^{-}\right)\right|}, \text {for } t \in\left(t_{1}, s_{1}\right], \\
\frac{\varepsilon}{2 \mathbf{E}_{\frac{1}{2}}\left(a s_{1}^{\frac{1}{2}}\right)} \cos \sqrt{s_{1}\left|x\left(t_{1}^{-}\right)\right|} E_{\frac{1}{2}}\left(a\left(t-s_{1}\right)^{\frac{1}{2}}\right), \text { for } t \in\left(s_{1}, t_{2}\right], \\
\vdots \\
\frac{\varepsilon}{2 \mathbf{E}_{\frac{1}{2}}\left(a t^{\frac{1}{2}}\right)} \cos \sqrt{t\left|x\left(t_{m-1}^{-}\right)\right|}, \text {for } t \in\left(t_{m-1}, s_{m}\right], \\
\frac{\varepsilon}{2 \mathrm{E}_{\frac{1}{2}}\left(a s_{m}^{\frac{1}{2}}\right)} \cos \sqrt{s_{m}\left|x\left(t_{m-1}^{-}\right)\right|} E_{\frac{1}{2}}\left(a\left(t-s_{m}\right)^{\frac{1}{2}}\right), \text { for } t \in\left(s_{m}, t_{m+1}\right], \\
\vdots
\end{array}\right.
$$


From Definition 2, choosing $\delta=\frac{\varepsilon}{\mathbf{E}_{\frac{1}{2}}\left(a \mathbf{T}^{\frac{1}{2}}\right)}$, we get that the solution of Equation (29) without impulses satisfies uniform and continuous dependence on the initial point. Choosing $2 \mathbf{M} \leq \frac{\varepsilon}{\mathbf{E}_{\frac{1}{2}}\left(a \mathbf{T}^{\frac{1}{2}}\right)}$, then the $\mathbf{h}_{i}(t, x)$ are uniformly bounded. Thus, the conditions $\left[H_{1}\right],\left[H_{4}\right]$, and $\left[H_{5}\right]$ hold, and all the assumptions in Remark 1 are satisfied.

The solutions of Equation (29) and the corresponding perturbation problem (with $x_{0}=1, \widetilde{x_{0}}=1.3, \mathbf{T}=$ $\frac{3}{2}, a=\frac{1}{5}, \varepsilon=\frac{1}{2}$ ) are shown in Figure 2 .

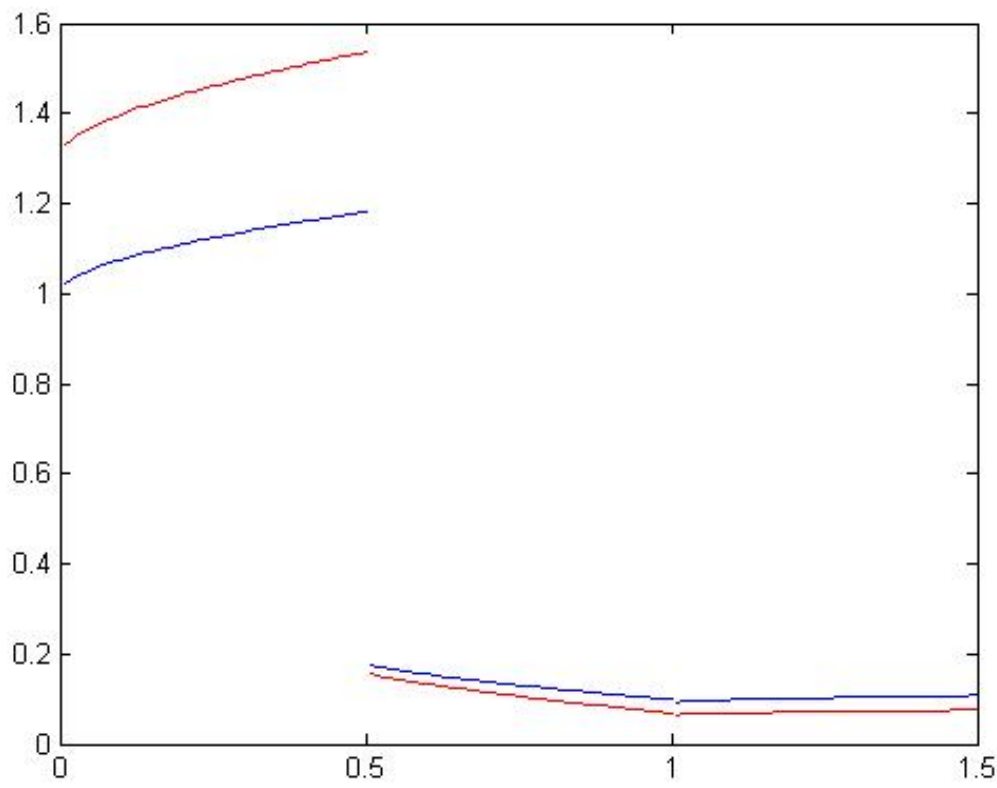

Figure 2. The blue line denotes the solution of Equation (29) and the red line denotes the corresponding perturbation problem.

Remark 2. Equations (27) and (29) are called non-instantaneous impulsive logistic models, which are motivated from the instantaneous impulsive logistic equations. For more details of the models, one can refer to Reference [12] (Section 4, therein).

\section{Conclusions}

In this paper, we presented the continuous dependence of the solutions to first order non-instantaneous IDEs with random impulse and junction points. Then, we extended the results to study the same problem for fractional order cases. The backward checking approach [34] (from the last subinterval to the first subinterval) is extended to differential and algebra equations and is used to prove the main results. The approach is different from Reference [13].

Author Contributions: The contributions of both authors (Y.C. and J.W.) are equal. All the main results and numerical examples were developed together.

Funding: This work was partially supported by the National Natural Science Foundation of China (Grant no. 11661016), Training Object of High Level and Innovative Talents of Guizhou Province ((2016)4006), Science and Technology Program of Guizhou Province ([2017]5788-10), and Major Research Project of Innovative Group in Guizhou Education Department ([2018]012).

Acknowledgments: The authors are grateful to the referees for their careful reading of the manuscript and their valuable comments. We also thank the editor.

Conflicts of Interest: The authors declare no conflict of interest. 


\section{References}

1. Chalishajar, D.N.; Malar, K.; Karthikeyan, K. Approximate controllability of abstract impulsive fractional neutral evolution equations with infinite delay in Banach spaces. Electron. J. Differ. Equ. 2013, 2013, 1-21.

2. Chalishajar, D.N.; Karthikeyan, K.; Anguraj, A. Existence results for impulsive perturbed partial neutral functional differential equations in Frechet spaces. Dyn. Contin. Discret. Impuls. Syst. Ser. Math. Anal. 2015, $22,25-45$.

3. Hernández, E.; O’Regan, D. On a new class of abstract impulsive differential equations. Proc. Am. Math. Soc. 2013, 141, 1641-1649. [CrossRef]

4. Wang, J.; Fečkan, M. A general class of impulsive evolution equations. Topol. Meth. Nonlinear Anal. 2015, 46, 915-934. [CrossRef]

5. Wang, J. Stability of noninstantaneous impulsive evolution equations. Appl. Math. Lett. 2017, 73, $157-162$. [CrossRef]

6. Chen, P.; Li, Y.; Yang, H. Perturbation method for nonlocal impulsive evolution equations. Nonlinear Anal. Hybrid Syst. 2013, 8, 22-30. [CrossRef]

7. Bai, L.; Nieto, J.J. Variational approach to differential equations with not instantaneous impulses. Appl. Math. Lett. 2017, 73, 44-48. [CrossRef]

8. Pierri, M.; O'Regan, D.; Rolnik, V. Existence of solutions for semi-linear abstract differential equations with not instantaneous impulses. Appl. Math. Comput. 2013, 219, 6743-6749. [CrossRef]

9. Pierri, M.; Henríquez, H.R.; Prokczyk, A. Global solutions for abstract differential equations with non-instantaneous impulses. Mediterr. J. Math. 2016, 34, 1685-1708. [CrossRef]

10. Hernández, E.; Pierri, M.; O’Regan, D. On abstract differential equations with non instantaneous impulses. Topol. Methods Nonlinear Anal. 2015, 46, 1067-1085.

11. Yang, D.; Wang, J. Non-instantaneous impulsive fractional-order implicit differential equations with random effects. Stoch. Anal. Appl. 2017, 35, 719-741. [CrossRef]

12. Yang, D.; Wang, J.; O'Regan, D. A class of nonlinear non-instantaneous impulsive differential equations involving parameters and fractional order. Appl. Math. Comput. 2018, 321, 654-671. [CrossRef]

13. Yang, D.; Wang, J.; O'Regan, D. Asymptotic properties of the solutions of nonlinear non-instantaneous impulsive differential equations. J. Frankl. Inst. 2017, 354, 6978-7011. [CrossRef]

14. Yang, D.; Wang, J.; O'Regan, D. On the orbital Hausdorff dependence of differential equations with non-instantaneous impulses. C. R. Acad. Sci. Paris Ser. I 2018, 356, 150-171. [CrossRef]

15. Abbas, S.; Benchohra, M. Uniqueness and Ulam stabilities results for partial fractional differential equations with not instantaneous impulses. Appl. Math. Comput. 2015, 257, 190-198. [CrossRef]

16. Muslim, M.; Kumar, A.; Fečkan, M. Existence, uniqueness and stability of solutions to second order nonlinear differential equations with non-instantaneous impulses. J. King Saud Univ. 2018, 30, 204-213. [CrossRef]

17. Colao, V.; Muglia, L.; Xu, H.K. An existence result for a new class of impulsive functional differential equations with delay. J. Math. Anal. Appl. 2016, 441, 668-683. [CrossRef]

18. Wang, J.; Fečkan, M. Non-Instantaneous Impulsive Differential Equations Basic Theory and Computation; IOP Publishing: Bristol, UK, 2018.

19. Wang, J.; Zhou, Y.; Lin, Z. On a new class of impulsive fractional differential equations. Appl. Math. Comput. 2014, 242, 649-657. [CrossRef]

20. Wang, J.; Fečkan, M.; Tian, Y. Stability analysis for a general class of non-instantaneous impulsive differential equations. Mediterr. J. Math. 2017, 14, 1-21. [CrossRef]

21. Wang, J.; Ibrahim, A.G.; O'Regan, D. Topological structure of the solution set for fractional non-instantaneous impulsive evolution inclusions. J. Fixed Point Theory Appl. 2018, 20, 1-25. [CrossRef]

22. Wang, J.; Ibrahim, A.G.; O'Regan, D.; Zhou, Y. Controllability for noninstantaneous impulsive semilinear functional differential inclusions without compactness. Indag. Math. 2018, 29, 1362-1392. [CrossRef]

23. Wang, J.; Ibrahim, A.G.; O'Regan, D. Hilfer type fractional differential switched inclusions with noninstantaneous impulsive and nonlocal conditions. Nonlinear Anal. Model. Contr. 2018, 23, 921-941. [CrossRef]

24. Wang, J.; Ibrahim, A.G.; O'Regan, D. Nonempty and compactness of solution set for fractional semilinear evolution inclusions with non-instantaneous impulses. Electr. J. Differ. Equ. 2019, 2019, 1-17. 
25. Liu, S.; Wang, J.; Shen, D.; O'Regan, D. Iterative learning control for parabolic partial differential inclusions with noninstantaneous impulses. Appl. Math. Comput. 2019, 350, 48-59. [CrossRef]

26. Luo, D.; Wang, J.; Shen, D. Learning formation control for fractional-order multi-agent systems. Math. Meth. Appl. Sci. 2018, 41, 5003-5014. [CrossRef]

27. Zhang, J.; Wang, J. Numerical analysis for a class of Navier-Stokes equations with time fractional derivatives. Appl. Math. Comput. 2018, 336, 481-489.

28. Zhu, B.; Liu, L.; Wu, Y. Local and global existence of mild solutions for a class of nonlinear fractional reaction-diffusion equation with delay. Appl. Math. Lett. 2016, 61, 73-79. [CrossRef]

29. Ren, L.; Wang, J.; Fečkan, M. Asymptotically periodic solutions for Caputo type fractional evolution equations. Fract. Calc. Appl. Anal. 2018, 21, 1294-1312. [CrossRef]

30. Wang, X.; Wang, J.; Shen, D.; Zhou, Y. Convergence analysis for iterative learning control of conformable fractional differential equations. Math. Meth. Appl. Sci. 2018, 41, 8315-8328. [CrossRef]

31. Wang, Y.; Liu, L.; Zhang, X.; Wu, Y. Positive solutions of a fractional semipositone differential system arising from the study of HIV infection models. Appl. Math. Comput. 2015, 258, 312-324.

32. Zhang, X.; Mao, C.; Liu, L.; Wu, Y. Exact iterative solution for an abstract fractional dynamic system model for bioprocess. Qual. Theory Dyn. Syst. 2017, 16, 205-222. [CrossRef]

33. Zhang, X.; Liu, L.; Wu, B.Y. Wiwatanapataphee, Nontrivial solutions for a fractional advection dispersion equation in anomalous diffusion. Appl. Math. Lett. 2017, 66, 1-8. [CrossRef]

34. Dishlieva, K. On the qualitative theory of differential equaitons with random impulsive moments. Int. J. Sci. Tech. Manag. 2015, 4, 172-180.

35. Kilbas, A.A.; Srivastava, H.M.; Trujillo, J.J. Theory and Applications of Fractional Differential Equations; Elsevier: Amsterdam, The Netherlands, 2006.

36. Bainov, D.; Simeonov, P. Integral Inequalities and Applications; Kluwer Academic Publishers: Dordrecht, The Netherlands, 1992 .

37. Ye, H.; Gao, J.; Ding, Y. A generalized Gronwall inequality and its application to a fractional differential equation. J. Math. Anal. Appl. 2007, 328, 1075-1081. [CrossRef]

(C) 2019 by the authors. Licensee MDPI, Basel, Switzerland. This article is an open access article distributed under the terms and conditions of the Creative Commons Attribution (CC BY) license (http:/ / creativecommons.org/licenses/by/4.0/). 